\title{
Efficient Synthesis of D-erythro-Sphingosine and D-erythro-Azidosphingosine from D-ribo- Phytosphingosine via a Cyclic Sulfate Intermediate
}

\author{
Sanghee Kim, ${ }^{*}$ Sukjin Lee, Taeho Lee, Hyojin Ko, and Deukjoon Kim
}

\section{Contents}

General Experimental Method

Experimental procedures for the synthesis of 5, 9 and $\mathbf{1 0 .}$

${ }^{1} \mathrm{H}$ NMR and ${ }^{13} \mathrm{C}$ NMR spectra of compound 5

${ }^{1} \mathrm{H}$ NMR and ${ }^{13} \mathrm{C}$ NMR spectra of compound 6

${ }^{1} \mathrm{H}$ NMR and ${ }^{13} \mathrm{C}$ NMR spectra of compound 7

S10-S11

${ }^{1} \mathrm{H}$ NMR and ${ }^{13} \mathrm{C}$ NMR spectra of compound $\mathbf{8}$

S12-S13

${ }^{1} \mathrm{H}$ NMR and ${ }^{13} \mathrm{C}$ NMR spectra of compound $\mathbf{3}$

S14-S15

${ }^{1} \mathrm{H}$ NMR and ${ }^{13} \mathrm{C}$ NMR spectra of compound $\mathbf{1}$

S16-S17

${ }^{1} \mathrm{H}$ NMR and ${ }^{13} \mathrm{C}$ NMR spectra of compound $\mathbf{1 0}$

S18-S19 
General. All chemicals were reagent grade and used as purchased. All reactions were performed under an inert atmosphere of dry argon or nitrogen using distilled dry solvents. Reactions were monitored by TLC analysis using silica gel 60 F-254 TLC plates. Melting points are uncorrected. Flash column chromatography was carried out on silica gel (230-400 mesh). Optical rotations were measured using sodium light (D line $589.3 \mathrm{~nm}) .{ }^{1} \mathrm{H}$ NMR and ${ }^{13} \mathrm{C}$ NMR spectra were recorded in $\delta$ units relative to deuterated solvent as internal reference at 300 and $75 \mathrm{MHz}$, respectively. IR spectra were measured on a Fourier Transform Infrared spectrometer. Mass spectra (MS) were recorded using fast atom bombardment $(\mathrm{FAB})$ or chemical ionization (CI). High resolution mass spectra (HRMS) were recorded using FAB or CI. 


\section{(2S,3S,4R)-2-Azido-1-(tert-butyldiphenylsilyoxy)octadecane-3,4-triol (5)}

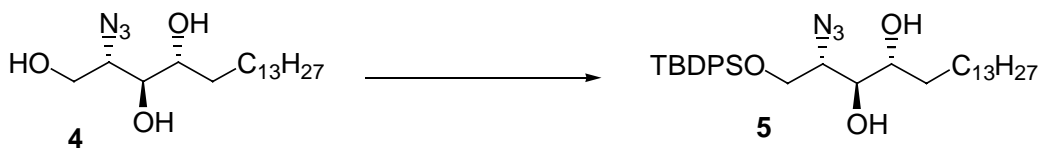

To a solution of 2-azido-phytosphingosine (1.25 g, $3.64 \mathrm{mmol})$ in $\mathrm{CH}_{2} \mathrm{Cl}_{2}(18 \mathrm{~mL})$

and DMF (4 mL) were added triethylamine (1.3 mL, 9.1 mmol), 4-DMAP (22 mg, 0.18

mmol) and TBDPS-Cl $(1.1 \mathrm{~mL}, 4.4 \mathrm{mmol})$ at $0{ }^{\circ} \mathrm{C}$. The reaction mixture was stirred at room temperature for $24 \mathrm{~h}$, and then diluted with EtOAc. The organic layer was washed with brine, dried over $\mathrm{Na}_{2} \mathrm{SO}_{4}$, concentrated. It was purified by column chromatography on silica gel (hexane/EtOAc, $5: 1)$ to give diol $5(1.84 \mathrm{~g}, 87 \%)$ as a colorless oil: $[\alpha]^{25}$ $+19.3\left(c\right.$ 1.3, $\left.\mathrm{CHCl}_{3}\right) ;{ }^{1} \mathrm{H} \mathrm{NMR}\left(\mathrm{CDCl}_{3}, 300 \mathrm{MHz}\right) \delta 0.89(\mathrm{t}, J=6.9 \mathrm{~Hz}, 3 \mathrm{H}), 1.09(\mathrm{~s}$, 9H), 1.27 (s, 22H), 1.41-1.60 (m, 2H), 2.17 (br s, 2H), 3.57 (m, 1H), 3.65-3.71 (m, 2H), $3.92(\mathrm{dd}, J=5.7,10.8 \mathrm{~Hz}, 1 \mathrm{H}), 4.04(\mathrm{dd}, J=3.9,10.8 \mathrm{~Hz}, 1 \mathrm{H}), 7.39-7.50(\mathrm{~m}, 6 \mathrm{H})$, 7.69-7.72 (m, 4H); ${ }^{13} \mathrm{C} \mathrm{NMR}\left(\mathrm{CDCl}_{3}, 75 \mathrm{MHz}\right) \delta 14.1,19.0,22.6,25.6,26.7,29.3$, 29.5, 29.6, 29.7, 31.6, 31.9, 63.4, 64.2, 72.3, 74.0, 127.8, 129.9, 132.45, 132.53, 135.5, 135.6; IR $\left(\mathrm{CHCl}_{3}\right) v_{\max } 3412,2099\left(\mathrm{~cm}^{-1}\right)$; HRMS (FAB) calcd for $\mathrm{C}_{34} \mathrm{H}_{54} \mathrm{O}_{3} \mathrm{~N}_{3} \mathrm{Si}$ $580.3934\left([\mathrm{M}-\mathrm{H}]^{-}\right)$, found 580.3939. 


\section{(2S,3S,4R)-2-Azido-1-(trityloxy)octadecane-3,4-triol (9)}

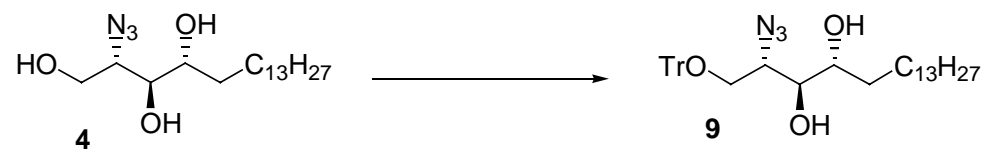

To a solution of the 2-azido-phytosphingosine 4 (2.19 g, $6.38 \mathrm{mmol})$ in pyridine (13 $\mathrm{mL})$ and $\mathrm{CH}_{2} \mathrm{Cl}_{2}(20 \mathrm{~mL})$ was added triphenyl methyl chloride $(2.14 \mathrm{~g}, 7.66 \mathrm{mmol})$. This reaction mixture was stirred under argon for $16 \mathrm{~h}$, then quenched with $0.5 \mathrm{M} \mathrm{HCl}$ $(15 \mathrm{~mL})$ and extracted with EtOAc twice. The combined organic layers were washed with brine, dried over $\mathrm{Na}_{2} \mathrm{SO}_{4}$, concentrated. It was purified by column chromatography on silica gel (hexane/EtOAc, 5:1) to give the diol $9(3.15 \mathrm{~g}, 84 \%)$ as a colorless oil: $[\alpha]_{\mathrm{D}}^{25}+14.6\left(c\right.$ 1.3, $\left.\mathrm{CHCl}_{3}\right)\left\{\right.$ lit. $^{18 \mathrm{~b}}[\alpha]_{\mathrm{D}}^{20}+8.9\left(c\right.$ 1.0, $\left.\mathrm{CHCl}_{3}\right)$, lit. $^{18 \mathrm{c}}[\alpha]_{\mathrm{D}}^{24}+11.9(c$ 0.9, $\left.\left.\mathrm{CHCl}_{3}\right)\right\} ;{ }^{1} \mathrm{H} \mathrm{NMR}\left(\mathrm{CDCl}_{3}, 300 \mathrm{MHz}\right) \delta 0.89(\mathrm{t}, J=6.9 \mathrm{~Hz}, 3 \mathrm{H}), 1.27-1.58(\mathrm{~m}, 24 \mathrm{H})$, 1.84 (br d, $J=4.2 \mathrm{~Hz}, 1 \mathrm{H}), 2.38(\mathrm{~d}, J=4.8 \mathrm{~Hz}, 1 \mathrm{H}), 3.43(\mathrm{dd}, J=5.4,10.2 \mathrm{~Hz}, 1 \mathrm{H})$, 3.53-3.58 (m, 2H), 3.63-3.68 (m, 2H), 7.24-7.36 (m, 9H), 7.46-7.50 (m, 6H); ${ }^{13} \mathrm{C}$ NMR $\left(\mathrm{CDCl}_{3}, 75 \mathrm{MHz}\right) \delta 14.0,22.6,25.6,29.3,29.5,29.6,31.5,31.8,62.3,63.5,72.1,74.0$, 87.6, 127.2, 127.9, 128.5, 143.4; IR $\left(\mathrm{CHCl}_{3}\right) v_{\max } 3416,2099\left(\mathrm{~cm}^{-1}\right)$. 


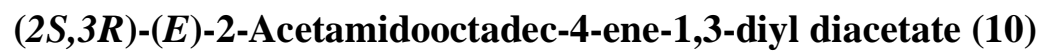

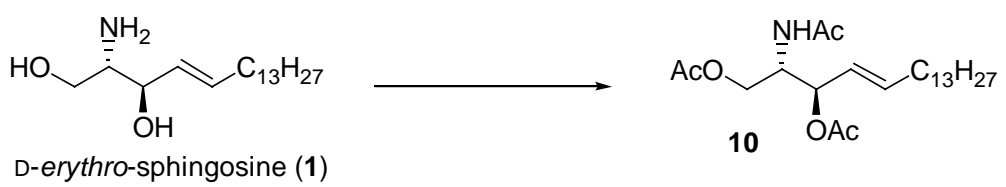

To a solution of sphingosine $\mathbf{1}(98 \mathrm{mg}, 0.33 \mathrm{mmol})$ in pyridine $(3 \mathrm{~mL})$ was added $\mathrm{Ac}_{2} \mathrm{O}(189 \mu \mathrm{L}, 2.0 \mathrm{mmol})$ at $0{ }^{\circ} \mathrm{C}$. After stirring at room temperature for $5 \mathrm{~h}$, this reaction mixture was poured into water and extracted with $\mathrm{CH}_{2} \mathrm{Cl}_{2}$ twice. The combined organic layers were washed with brine, dried over $\mathrm{MgSO}_{4}$ and concentrated. The crude product was purified by silica gel column chromatography (hexane/EtOAc, 1:1) to give sphingosine triacetate $\mathbf{1 0}(126 \mathrm{mg}, 90 \%)$ as a white solid; mp $102-104{ }^{\circ} \mathrm{C}$ (lit. ${ }^{6 \mathrm{a}} \mathrm{mp} 100$ $102{ }^{\circ} \mathrm{C}$, lit. ${ }^{22 \mathrm{a}} \mathrm{mp} 101-102{ }^{\circ} \mathrm{C}$, lit. $\left.{ }^{22 \mathrm{~b}} \mathrm{mp} 104.6-106.0{ }^{\circ} \mathrm{C}\right) ;[\alpha]^{24} \mathrm{D}-14.4\left(c 1.2, \mathrm{CHCl}_{3}\right)$ (Optical rotation values of sphingosine triacetate $\mathbf{1 0}$ range from -13.0 to -13.2 . See: refs 6a, 8 and 22a); ${ }^{1} \mathrm{H}$ NMR $\left(\mathrm{CDCl}_{3}, 300 \mathrm{MHz}\right) \delta 0.87(\mathrm{t}, J=6.6 \mathrm{~Hz}, 3 \mathrm{H}), 1.25(\mathrm{~s}, 22 \mathrm{H})$, $1.98(\mathrm{~s}, 3 \mathrm{H}), 2.01-2.04(\mathrm{~m}, 2 \mathrm{H}), 2.056(\mathrm{~s}, 3 \mathrm{H}), 2.062(\mathrm{~s}, 3 \mathrm{H}), 4.03(\mathrm{dd}, J=3.9,11.4 \mathrm{~Hz}$, 1H), $4.29(\mathrm{dd}, J=6.0,11.4 \mathrm{~Hz}, 1 \mathrm{H}), 4.43(\mathrm{~m}, 1 \mathrm{H}), 5.27$ (app. t, $J=6.5 \mathrm{~Hz}, 1 \mathrm{H}), 5.38$ (dd, $J=7.5,15.3 \mathrm{~Hz}, 1 \mathrm{H}), 5.66$ (br d, $J=9.0 \mathrm{~Hz}, 1 \mathrm{H}), 5.78(\mathrm{td}, J=6.9,15.3 \mathrm{~Hz}, 1 \mathrm{H})$;

${ }^{13} \mathrm{C} \mathrm{NMR}\left(\mathrm{CDCl}_{3}, 75 \mathrm{MHz}\right) \delta 14.1,20.8,21.1,22.7,23.3,28.9,29.1,29.3,29.4,29.57$, 29.64, 31.9, 32.3, 50.7, 62.6, 73.8, 124.1, 137.4, 169.6, 170.0, 170.9; IR $\left(\mathrm{CHCl}_{3}\right) v_{\max }$ 3290, 1736, 1657, $1552\left(\mathrm{~cm}^{-1}\right)$; HRMS (FAB) calcd for $\mathrm{C}_{24} \mathrm{H}_{44} \mathrm{O}_{5} \mathrm{~N} 426.3219\left([\mathrm{M}+\mathrm{H}]^{+}\right)$, found 426.3230 . 


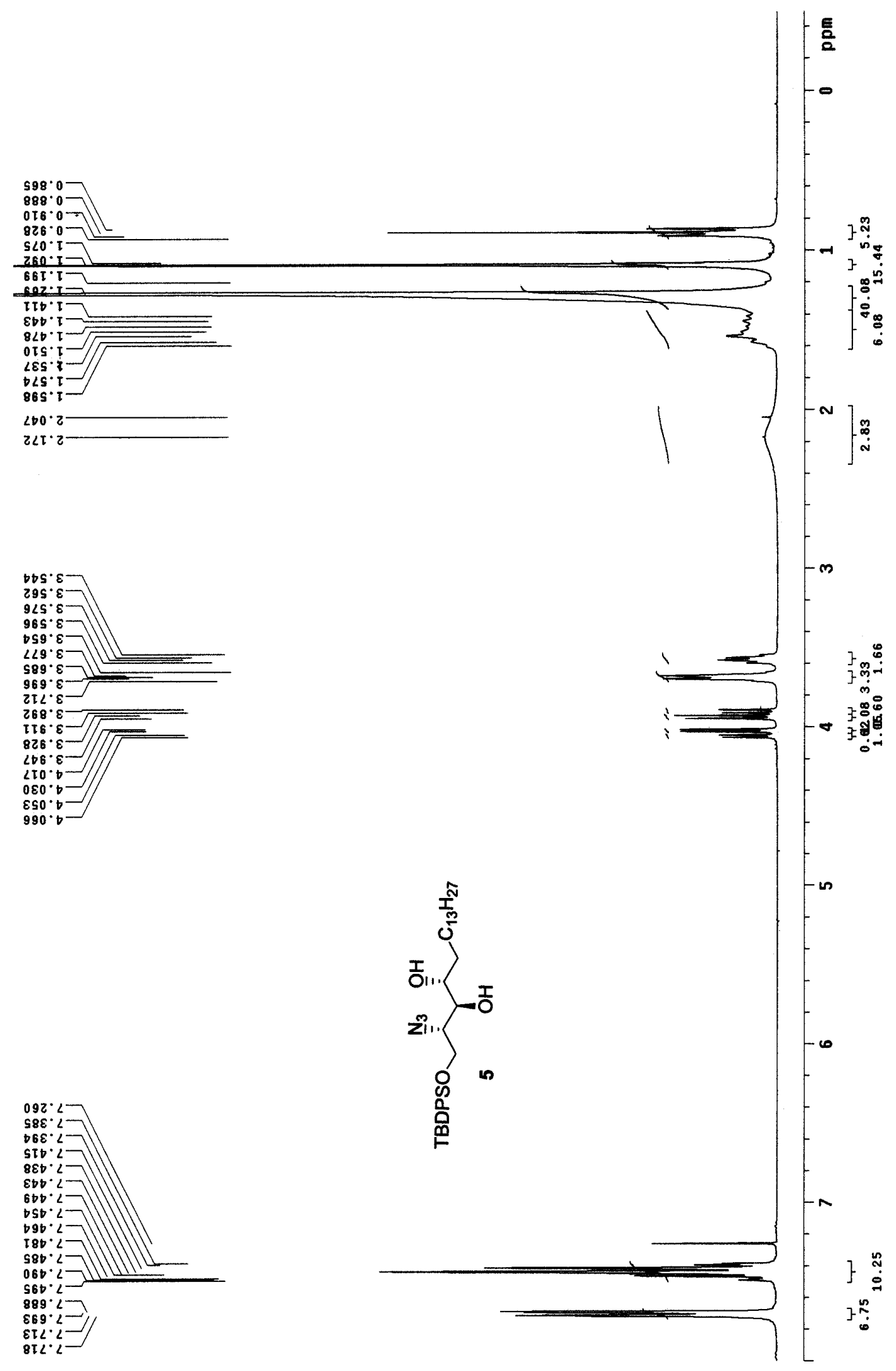



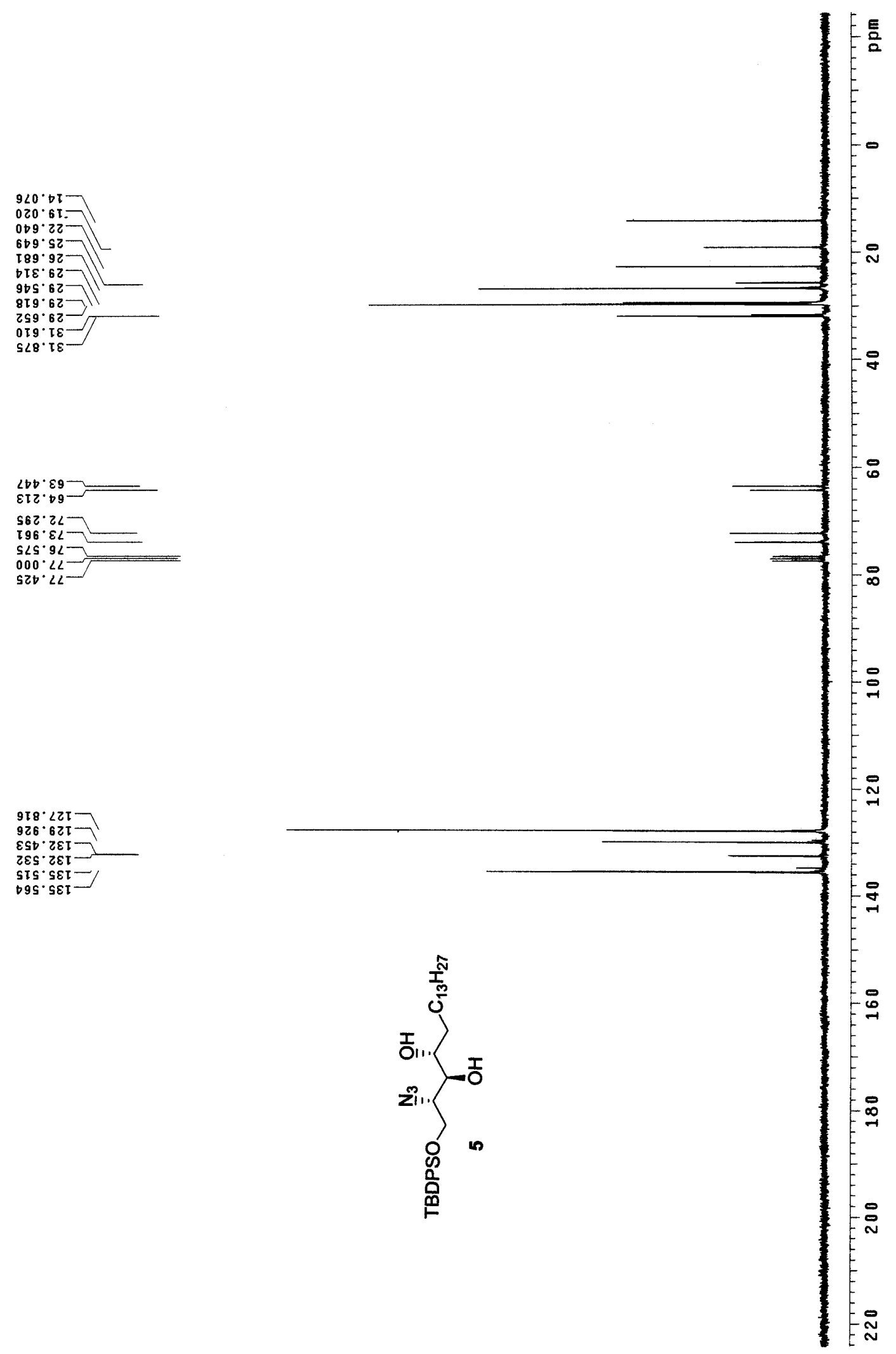


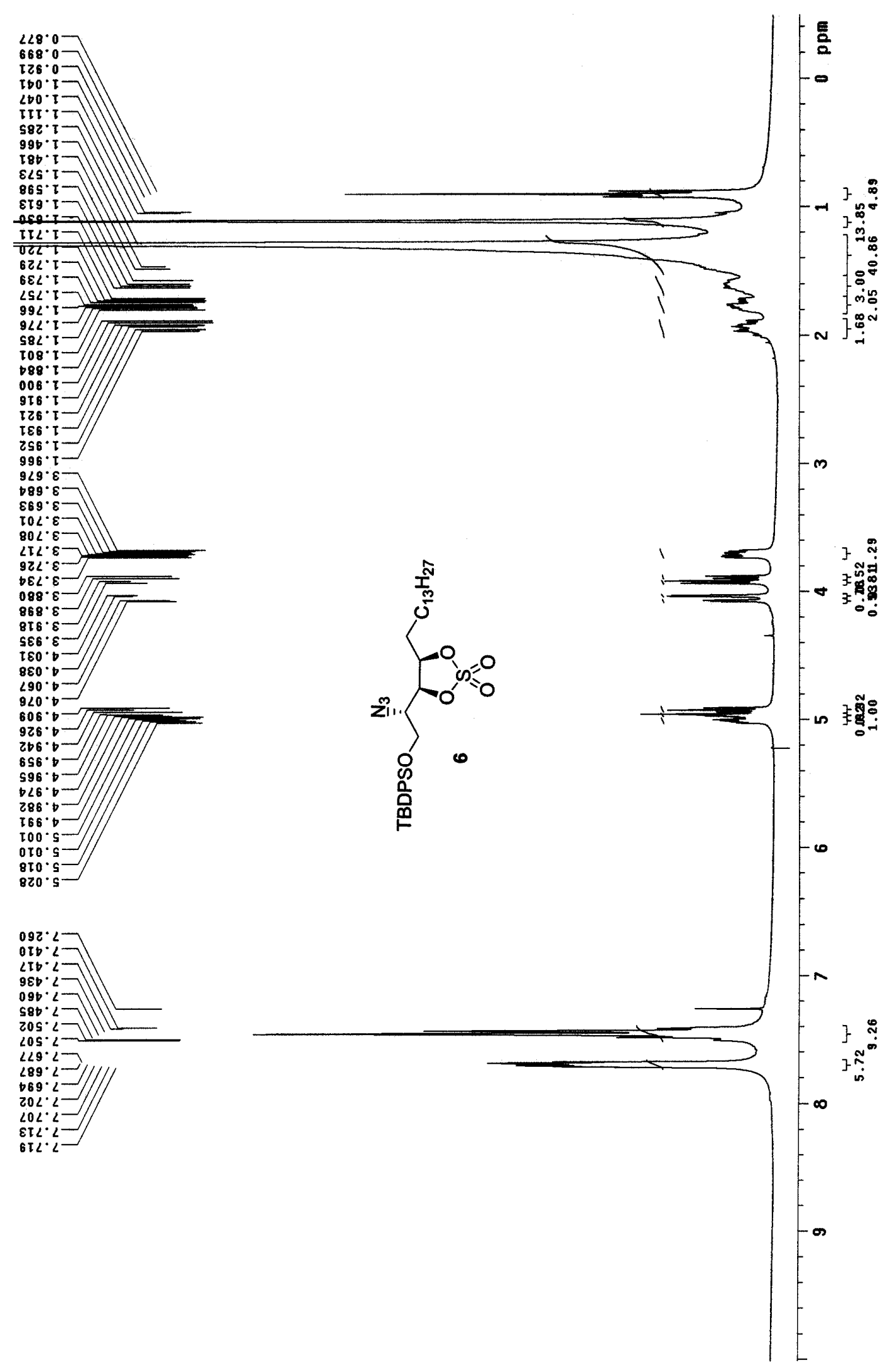



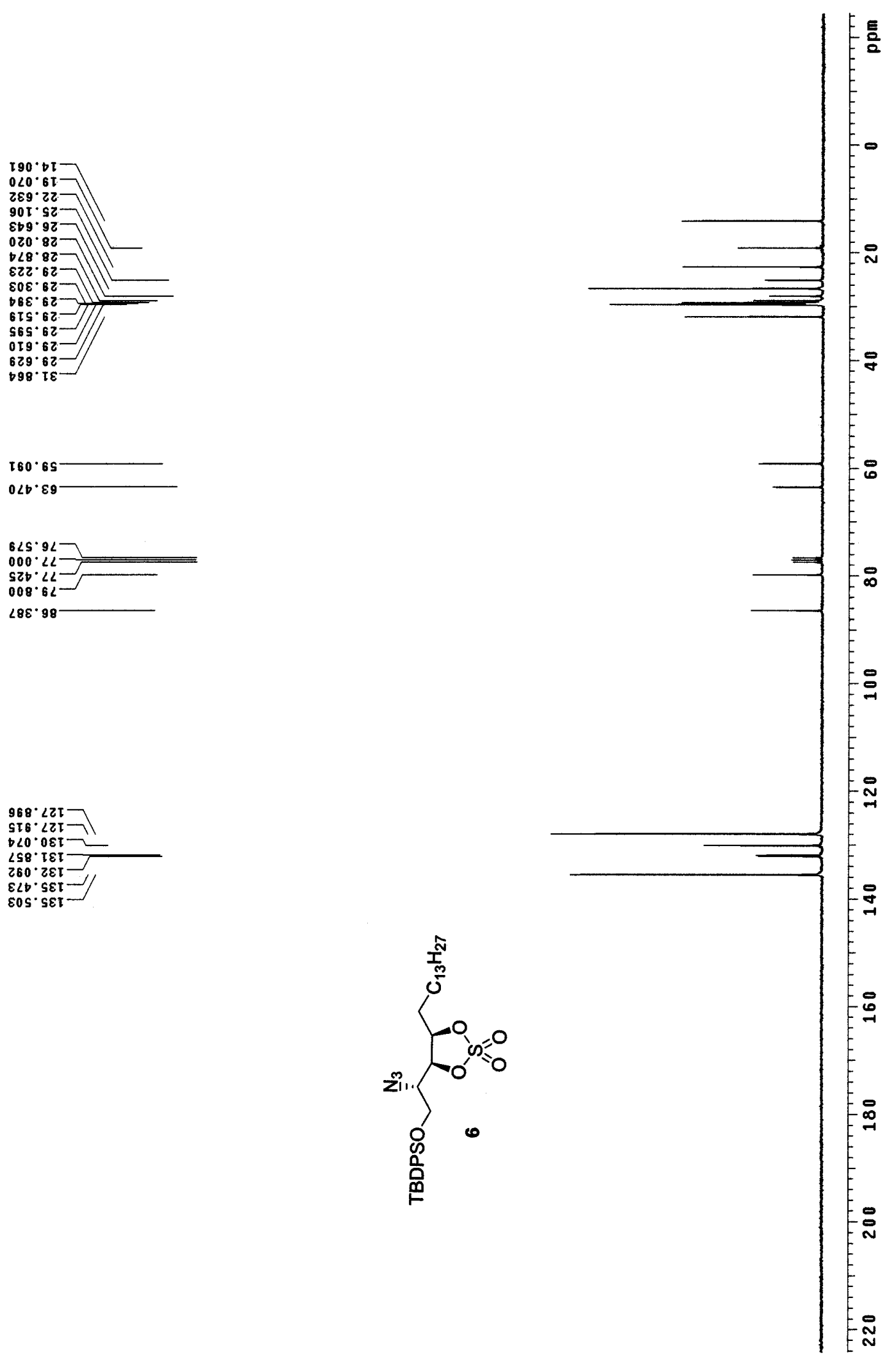


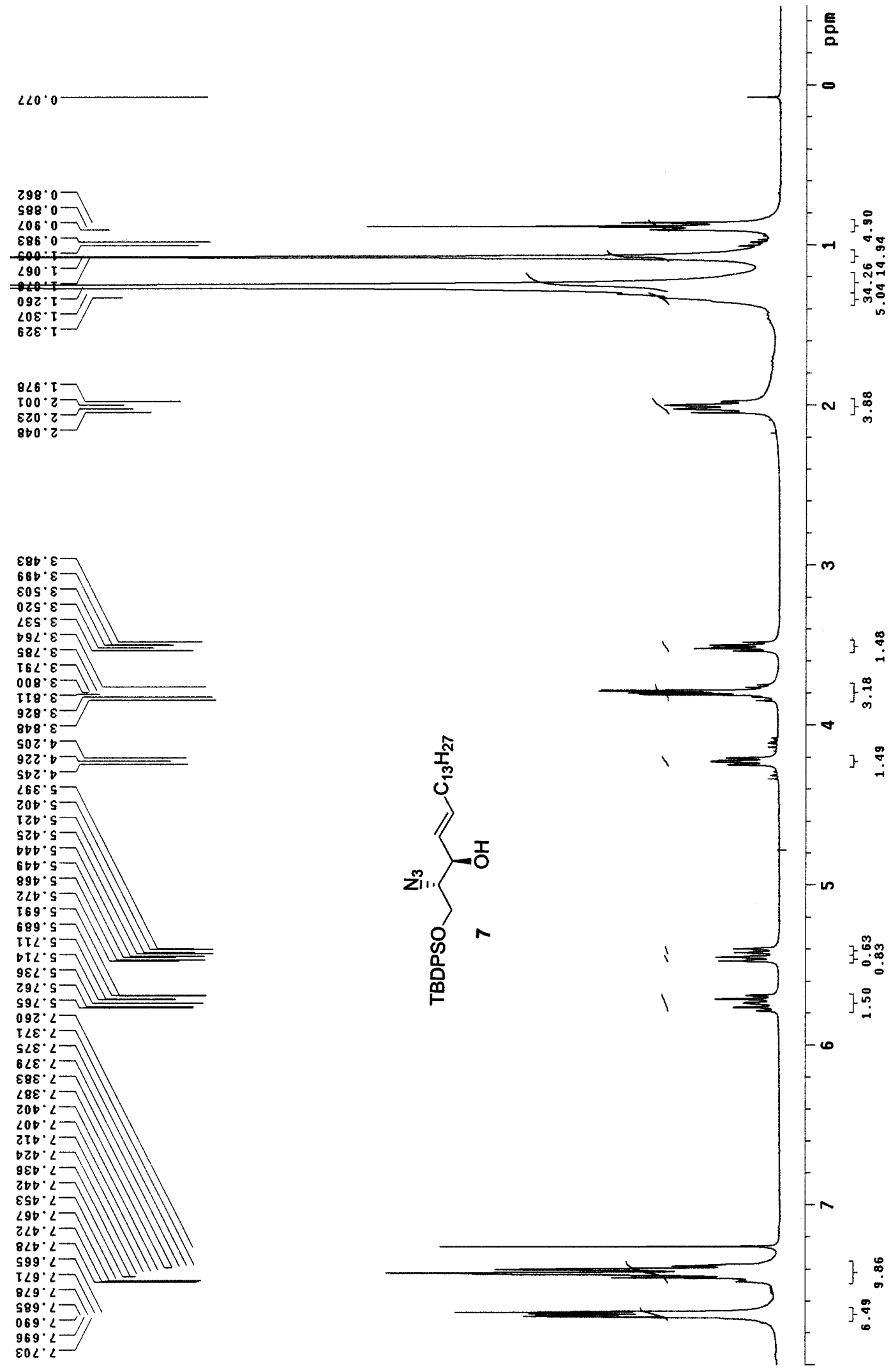



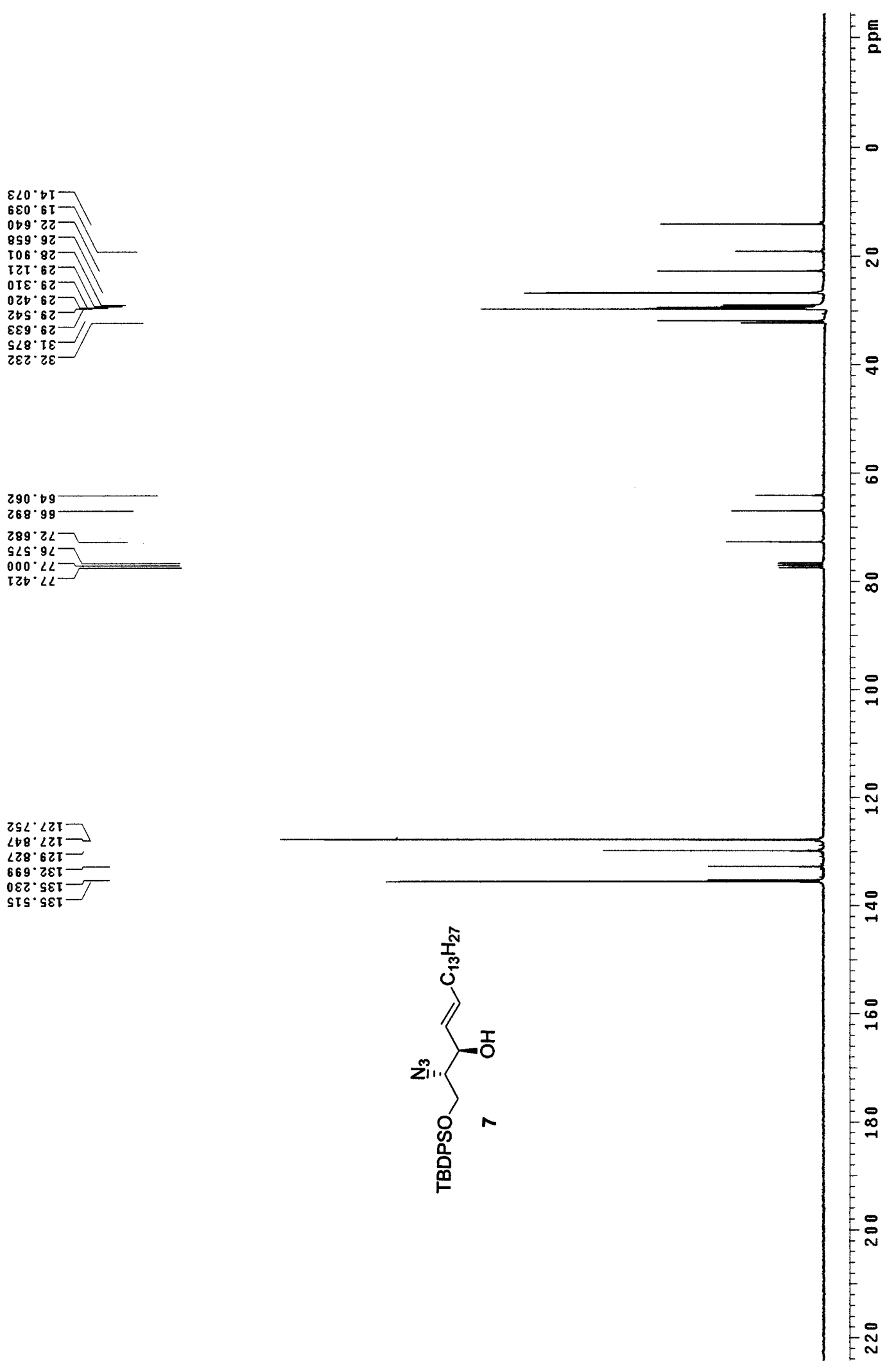


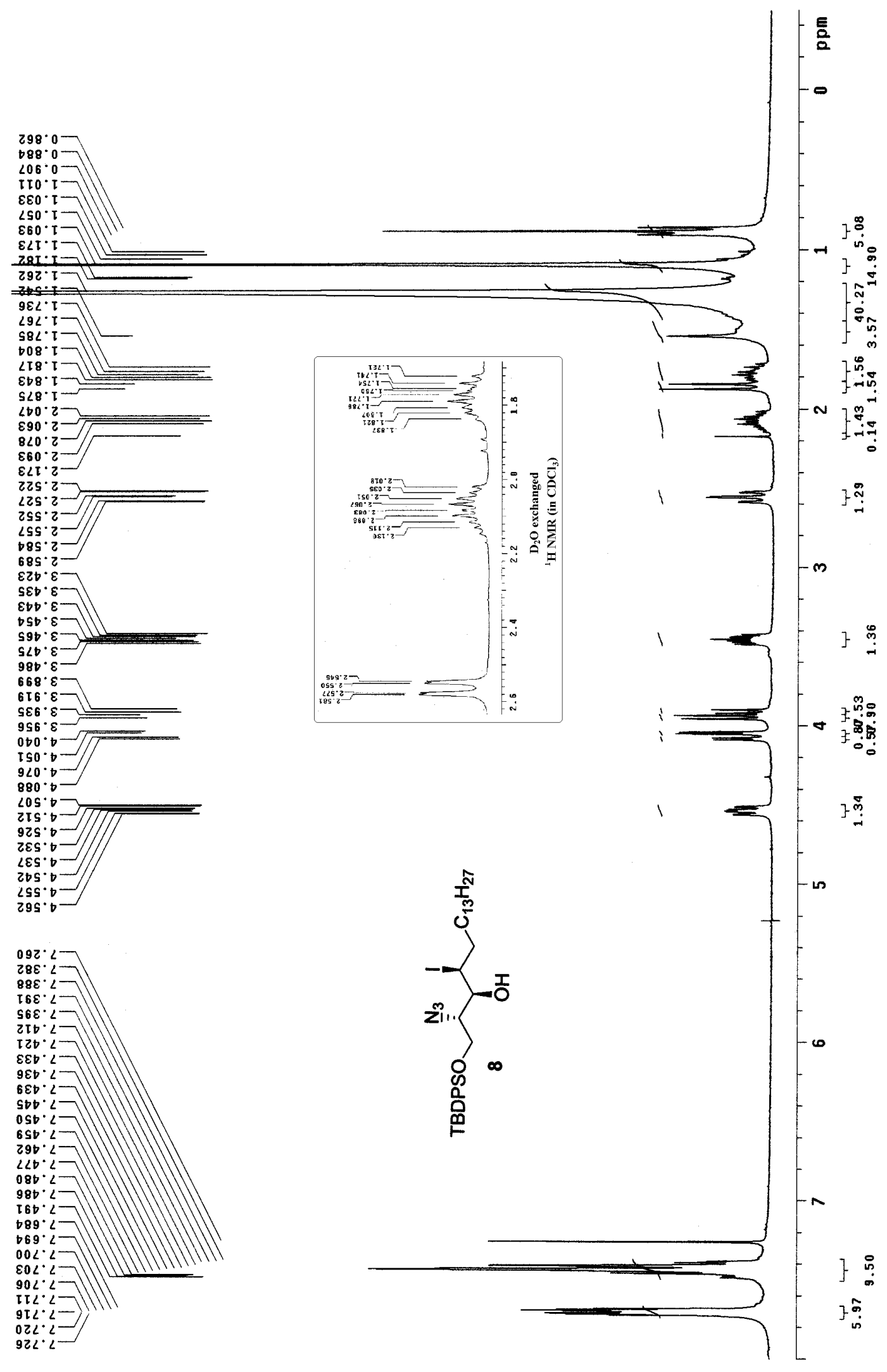



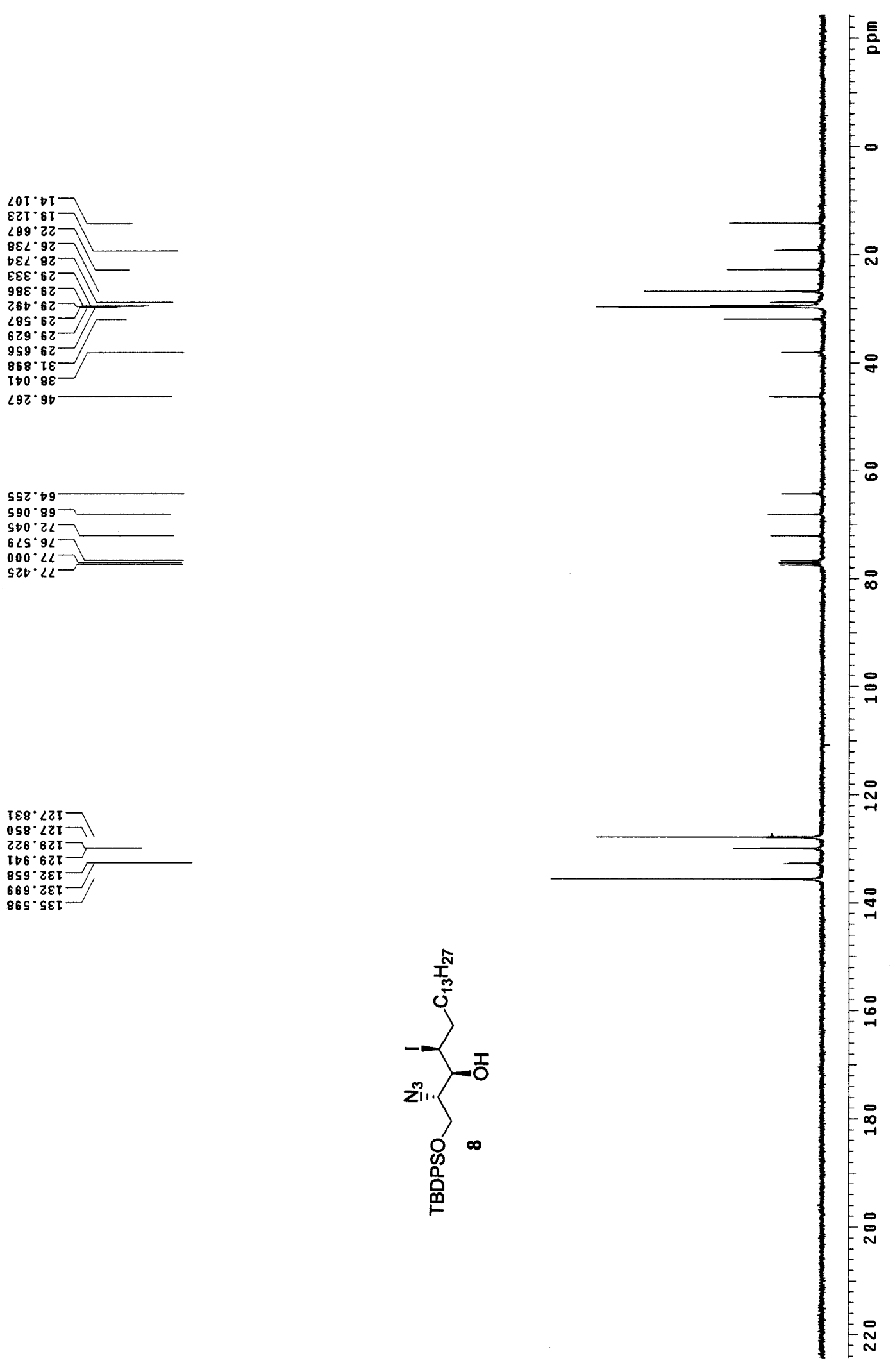


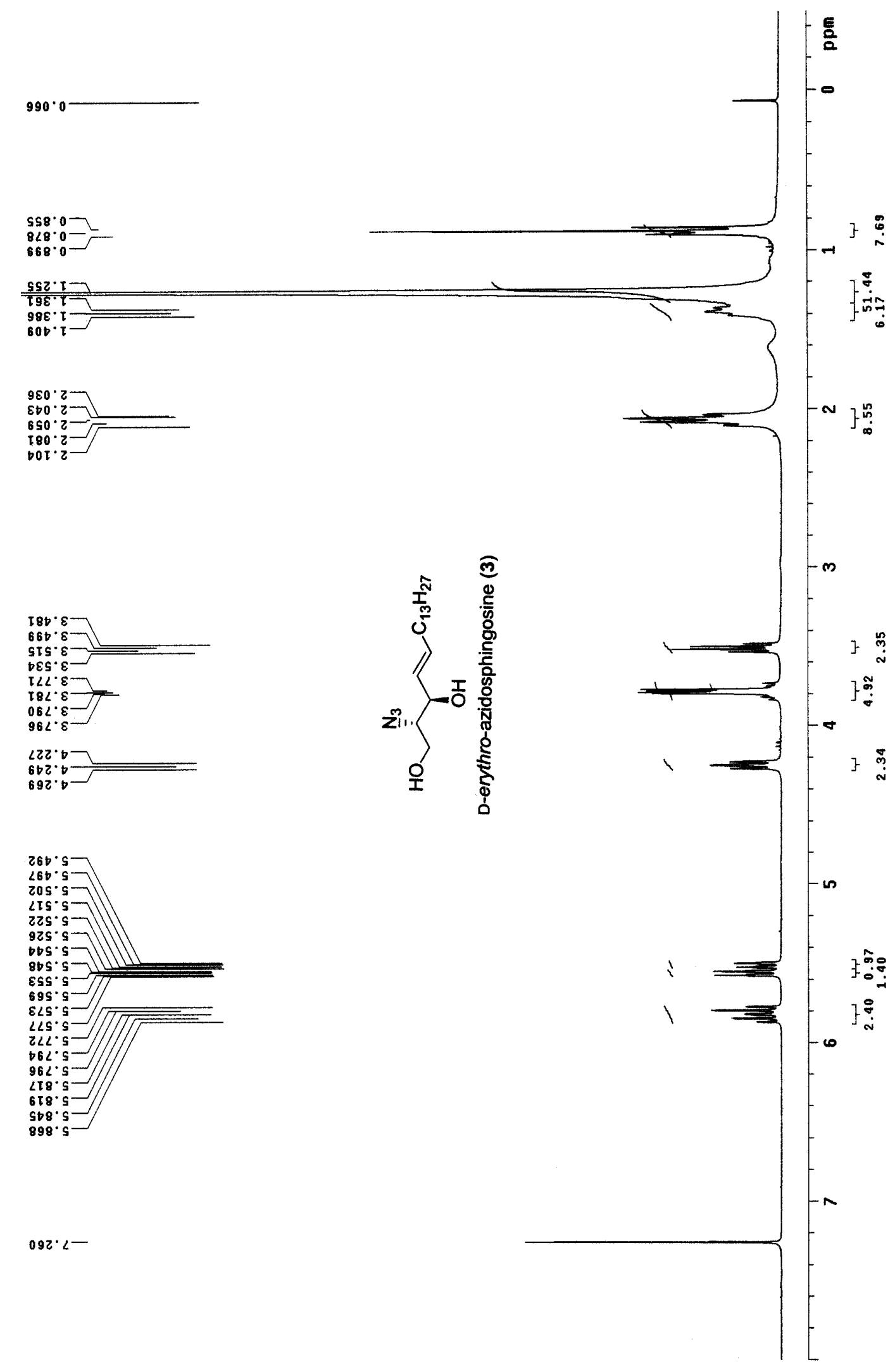



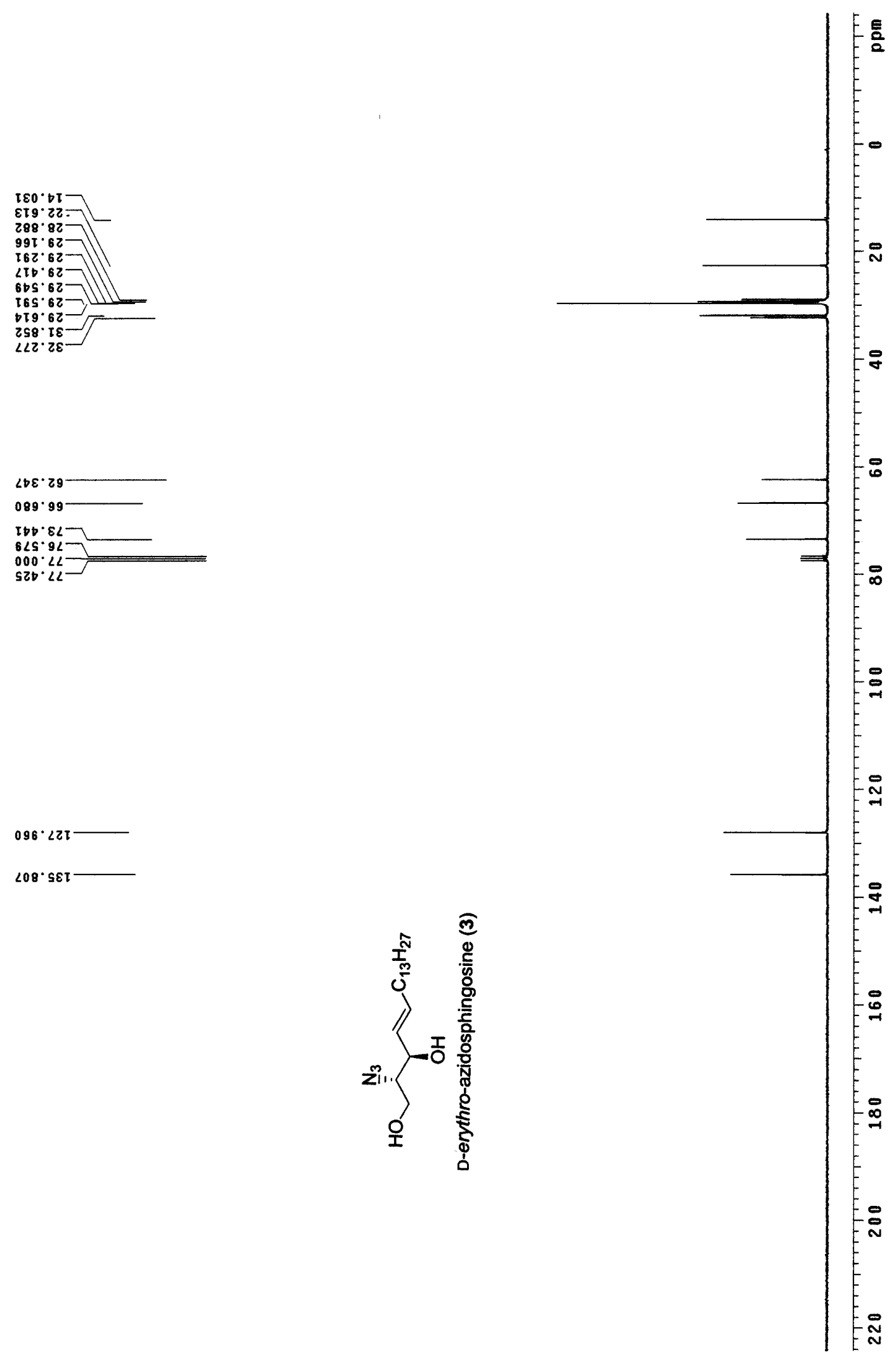


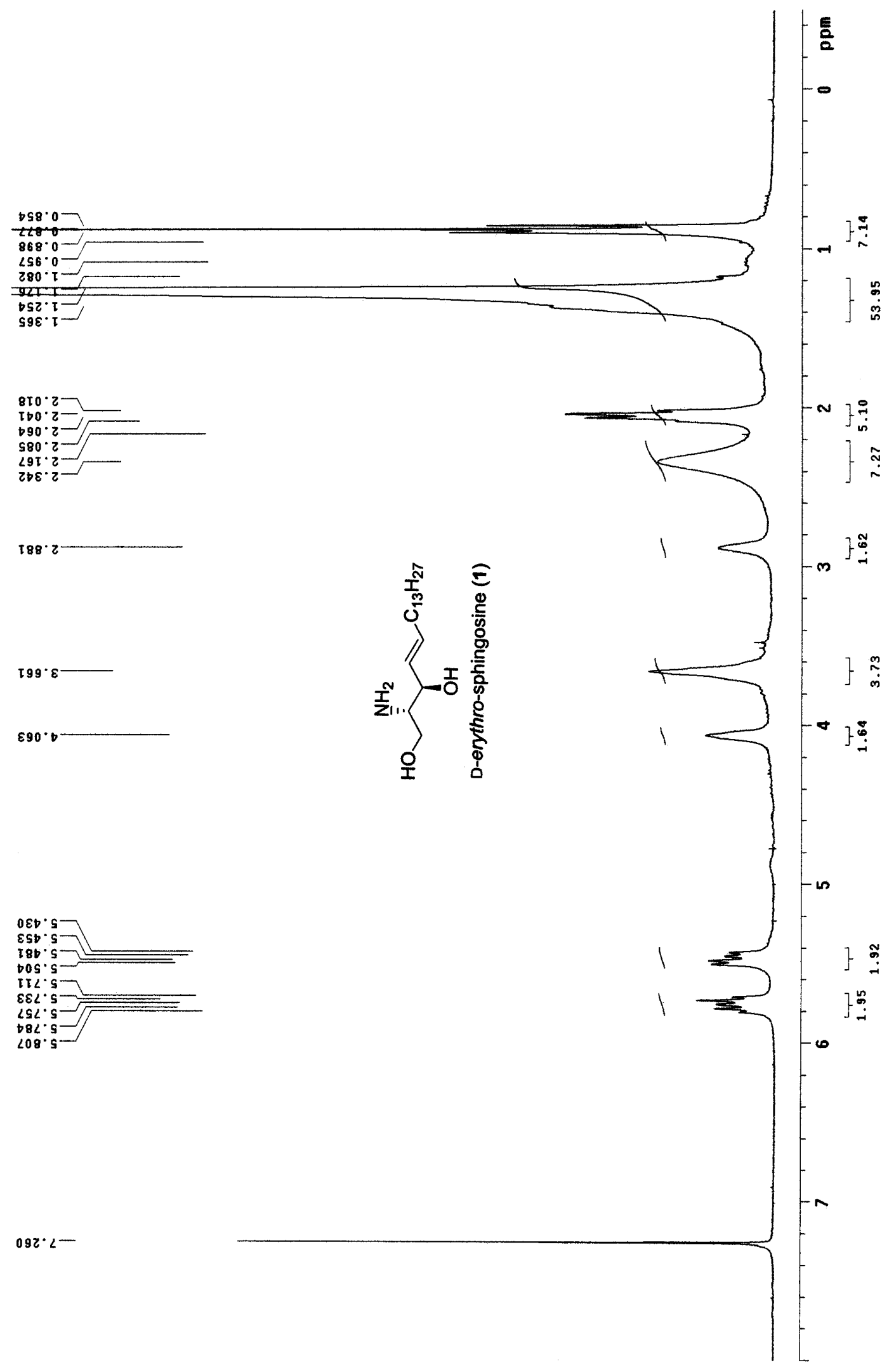




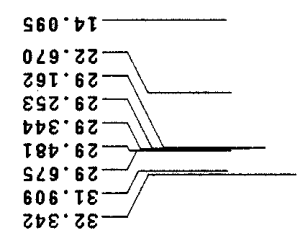

$26 I \cdot 9 S$

$069.89 \square$

$\varepsilon<0^{\circ} S L$

$6 \angle S^{\circ} 9 L$

$S 20 \cdot 2 L$

$660.621+$

$\varepsilon \varepsilon\llcorner\cdot \sqcup \varepsilon \tau$

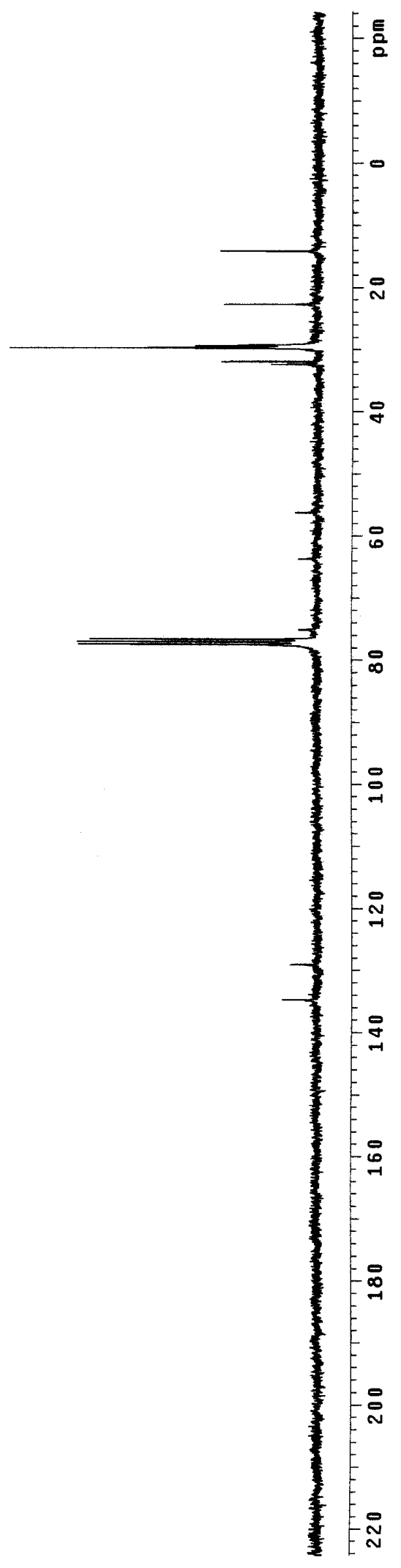




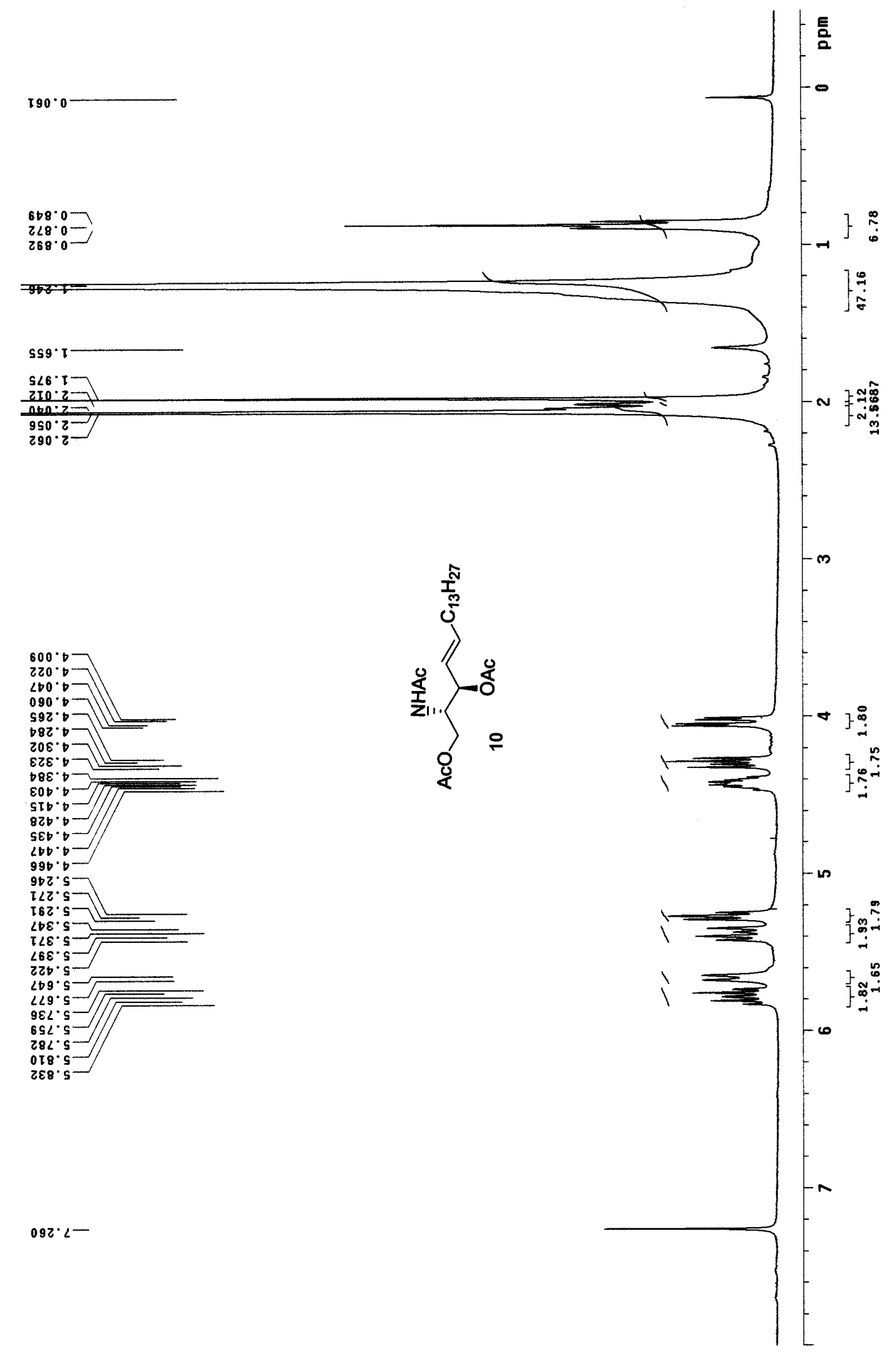



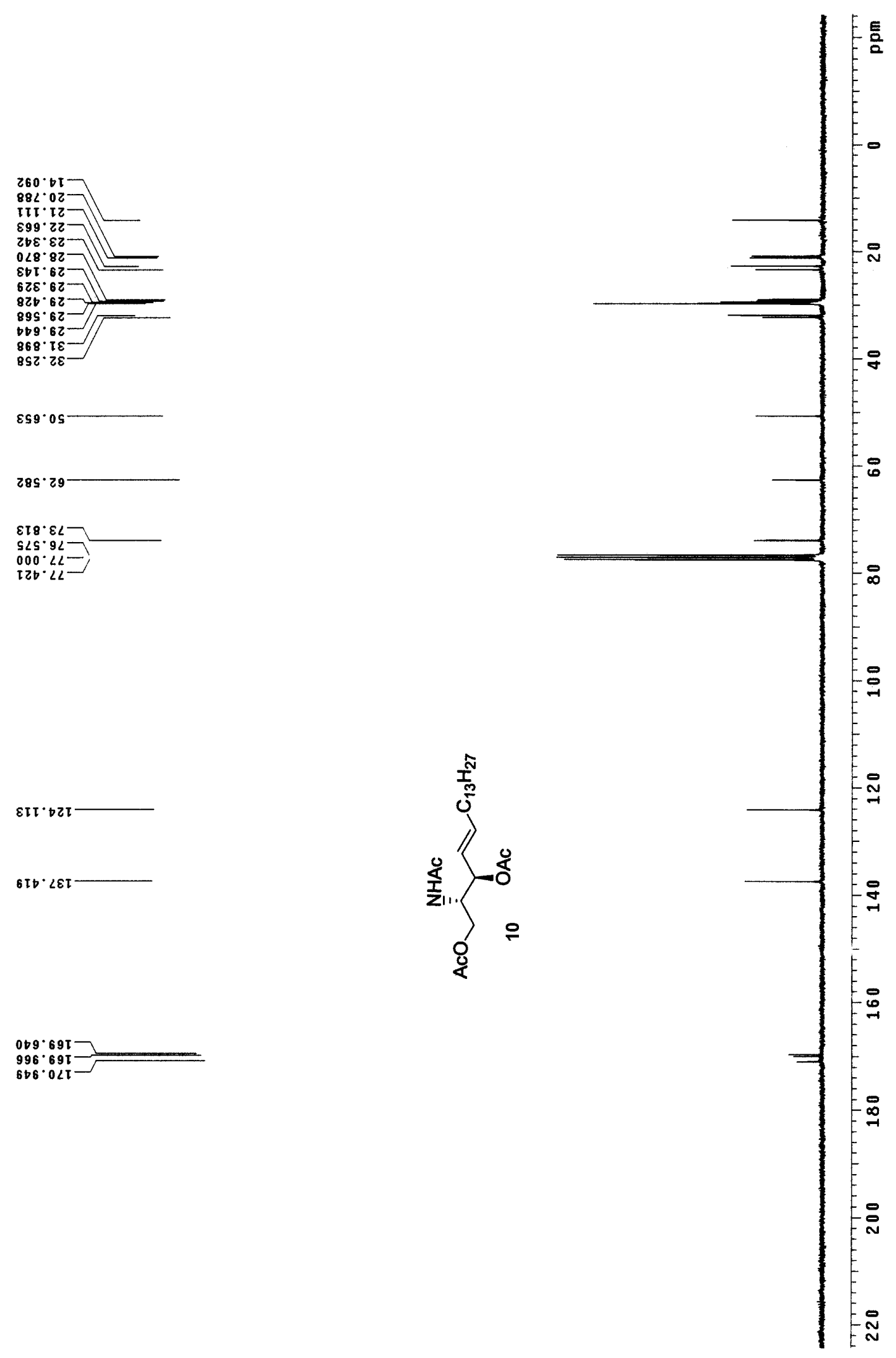

$\varepsilon \tau \tau \cdot \bullet \tau I$

$6 I b^{*} \angle \varepsilon I \longrightarrow$

696.621 